\title{
XTE J1946+274 = GRO J1944+26: An Enigmatic Be/X-ray Binary
}

\author{
Colleen. A. Wilson ${ }^{1}$, Mark H. Finger ${ }^{2}$ \\ SD 50 Space Science Research Center, National Space Science and Technology Center, 320 Sparkman \\ Drive, Huntsville, AL 35805 \\ colleen.wilson-hodge@msfc.nasa.gov \\ M.J. Coe \\ Dept. of Physics and Astronomy, The University, Southampton, SO17 1BJ, England \\ Ignacio Negueruela ${ }^{3}$ \\ Observatoire de Strasbourg, 11 rue de l'Université, 67000 Strasbourg, France
}

\begin{abstract}
XTE J1946+274 = GRO J1944+26 is a 15.8 s Be/X-ray pulsar discovered simultaneously in 1998 September with the Burst and Transient Source Experiment (BATSE) on the Compton Gamma Ray Observatory (CGRO) and the All-Sky Monitor (ASM) on the Rossi X-ray Timing Explorer (RXTE). Here we present new results from BATSE and RXTE including a pulse timing analysis, spectral analysis, and evidence for an accretion disk. Our pulse timing analysis yielded an orbital period of 169.2 days, a moderate eccentricity of 0.33 , and implied a mass function of $9.7 \mathrm{M}_{\odot}$. We observed evidence for an accretion disk, a correlation between measured spin-up rate and flux, which was fitted to obtain a distance estimate of $9.5 \pm 2.9 \mathrm{kpc}$. XTE J1946+274 remained active from 1998 September - 2001 July, undergoing 13 outbursts that were not locked in orbital phase. Comparing RXTE PCA observations from the initial bright outburst in 1998 and the last pair of outbursts in 2001, we found energy and intensity dependent pulse profile variations in both outbursts and hardening spectra with increasing intensity during the fainter 2001 outbursts. In 2001 July, optical $\mathrm{H} \alpha$ observations indicate a density perturbation appeared in the Be disk as the X-ray outbursts ceased. We propose that the equatorial plane of the $\mathrm{Be}$ star is inclined with respect to the orbital plane in this system and that this inclination may be a factor in the unusual outburst behavior of the system.
\end{abstract}

Subject headings: accretion—stars:pulsars:individual:(XTE J1946+274, GRO J1944+26)—X-rays: binaries

\footnotetext{
${ }^{1}$ NASA's Marshall Space Flight Center

${ }^{2}$ Universities Space Research Association

${ }^{3}$ Now at: Dpto. de Física, Ingeniería de Sistemas y Teoría de la Señal, Universidad de Alicante, Apdo. de Correos 99, E03080, Alicante, Spain
}

\section{Introduction}

$\mathrm{Be} / \mathrm{X}$-ray binaries are the most common type of accreting X-ray pulsar systems. They consist of a pulsar and a Be (or Oe) star, a main sequence star of spectral type B (or O) that shows Balmer emission lines (See e.g., Slettebak 1988 and Coe 2000 for reviews.) The line emission is believed to be associated with an equatorial outflow of material 
expelled from the rapidly rotating Be star which probably forms a quasi-Keplerian disk near the Be star (Hanuschik 1996; Quirrenbach et al. 1997). $\mathrm{X}$-ray outbursts are produced when the pulsar interacts with this disk. Be/X-ray binaries typically show two types of outburst behavior: (a) giant outbursts (or type II), characterized by high luminosities $\left(L_{\mathrm{X}} \gtrsim 10^{37} \mathrm{ergs} \mathrm{s}^{-1}\right)$ and high spin-up rates (i.e., a significant increase in pulse frequency) and (b) normal outbursts (or type I), characterized by lower luminosities $\left(L_{\mathrm{X}} \sim 10^{36}-10^{37} \mathrm{ergs}\right.$ $\mathrm{s}^{-1}$ ), low spin-up rates (if any), and recurrence at the orbital period (Stella, White, \& Rosner 1986; Bildsten et al. 1997). As a population Be/X-ray binaries show a correlation between their spin and orbital periods (Corbet 1986; Waters \& van Kerkwijk 1989).

On 1998 September 8 a 15.8 s pulsar, which was designated GRO J1944+26, was discovered with the Burst and Transient Source Experiment (Fishman et al. 1989, BATSE) on the Compton Gamma Ray Observatory (CGRO). GRO J1944+26 was localized to a $5^{\circ} \times 8^{\circ}$ error box (Wilson et al. 1998). At the same time, the All-Sky Monitor (Levine et al. 1996, ASM) on the Rossi Xray Timing Explorer (RXTE) discovered a new source which they localized to a $6^{\prime} \times 26^{\prime}$ error box and designated XTE J1946+274 (Smith \& Takeshima 1998). Subsequent observations with the Proportional Counter Array (Jahoda et al. 1996, PCA) also on the RXTE on 1998 September 16 revealed $15.8 \mathrm{~s}$ pulsations, confirming that BATSE and RXTE were seeing the same object (Smith \& Takeshima 1998). Scanning observations with the PCA further improved the position to a $2.4^{\prime}$ error circle (Takeshima \& Chakrabarty 1998). Observations with BeppoSAX further improved the X-ray position to R.A. $=19 h 45 \mathrm{~m} 38 \mathrm{~s}$, Decl. $=+27^{\circ} 21^{\prime} .5$ (equinox 2000.0; error radius $1^{\prime}$ at $95 \%$ confidence) (Campana, Israel, \& Stella 1998). XTE J1946+274 lies in the error box of the Ariel 5 transient 3A $1942+274$; however, there is a non-negligible probability that this is a chance association (Campana, Israel, \& Stella 1999).

A likely optical counterpart has been identified as an optically faint $B \sim 18.6 \mathrm{mag}$, bright infrared $(H \sim 12.1)$ Be star at R.A. $=19 h 45 m 39 s$, Decl. $=27^{\circ} 22^{\prime} 00^{\prime \prime}$, which lies in the refined BeppoSAX error circle(Verrecchia et al. 2002). Spectroscopic and photometric data indicate a B0-1IV-Ve star at
8-10 kpc. The counterpart's mass is not well constrained, but is expected to be in the $10-16 M_{\odot}$ range. Two previously proposed candidates (Israel, Polcaro, \& Covino 1998; Ghavamian \& Garcia 1998) lie $10^{\prime \prime}$ outside the BeppoSAX error circle.

Using data from the 1998 outburst taken with the PCA and the High Energy X-ray Timing Experiment (Rothschild et al. 1998, HEXTE) on RXTE, Heindl et al. (2002) revealed a cyclotron resonance scattering feature or cyclotron line near $35 \mathrm{keV}$. This feature implies a magnetic field strength of $3.1(1+z) \times 10^{12} \mathrm{G}$, where $z$ is the gravitational redshift of the emission region. Using about 1 year of RXTE ASM 2-10 keV flux measurements, beginning with the 1998 outburst, Campana, Israel, \& Stella (1999) observed 5 outbursts in which they found evidence of an $\sim 80$ day modulation in the outburst flux. They found that the first outburst in September 1998 was markedly different in rise and decay timescales than the 4 following outbursts and based on this suggested that the first outburst might be classified as a giant outburst. XTE J1946+274 was observed with the Indian X-ray Astronomy Experiment (Paul et al. 2001, IXAE) 1999 September 18-30 (MJD 51408-51421) and 2000 June 28-July 7 (MJD 51723-51733). They detected $15.8 \mathrm{~s}$ pulsations in the 2-6 and 6-18 keV bands, with similar double peaked profiles in both observations. The pulse period history was consistent with a constant intrinsic spin-up plus an eccentric orbit. The reported pulse periods were $P=15.78801(04) \mathrm{s}$ and on MJD 51445.0 and $P=15.76796(18)$ s on MJD 51727.5.

XTE J1946+274 was active from 1998 September through 2001 July, when it dropped below the detection threshold of the RXTE PCA. As of 2002 July, no additional outbursts have been seen with the RXTE ASM. Table 1 lists the dates and instruments observing XTE J1946+274's location. In this paper we will present results from observations of XTE J1946+274 with BATSE and $R X T E$, including histories of pulse frequency and total flux for XTE J1946+274. From the pulse frequency history, we derive an orbital solution. Applying this orbital solution, we will investigate the observed correlation between spin-up torques and observed flux and discuss implications for the accretion mechanism. We will describe the unusual 
orbital phasing of the outbursts. Using data from the RXTE PCA and HEXTE, we will investigate spectral variations in the initial bright outburst and the last 2 outbursts. We compare our X-ray results to optical $\mathrm{H} \alpha$ observations.

\section{Analysis and Results}

\subsection{Frequency Search}

To determine pulse frequencies for XTE J1946+274, we performed a grid search over a range of candidate frequencies using data from the Large Area Detectors on BATSE(Fishman et al. 1989). This search technique is described in detail in Finger et al. (1999) and Wilson-Hodge (1999). We will briefly summarize the technique here. Variations of this technique, including searches in pulse period, have been widely used (Buccheri et al. 1983; Bildsten et al. 1997, for example).

The pulsar search technique consists of 3 steps (1) data selection and combination, (2) $20-50 \mathrm{keV}$ pulse profile estimates, and (3) a grid search in frequency. First, the BATSE DISCLA channel 1 (20$50 \mathrm{keV}, 1 \mathrm{~s}$ time resolution) data were selected for which the source was visible, the high voltage was on, the spacecraft was outside the South Atlantic Anomaly, and no electron precipitation events or other anomalies were flagged by the BATSE mission operations team. The count rates were combined over the 4 LADs viewing XTE J1946+274, using weights optimized for an exponential energy spectrum, $f(E)=A \exp (-E / k T)$ with temperature $k T=12 \mathrm{keV}$, and grouped into $\approx 300 \mathrm{~s}$ segments. A segment length of $300 \mathrm{~s}$ was used because it was short enough that the background was well-fitted by a quadratic model. The segment boundaries were chosen to avoid Earth occultation steps from bright sources(Wilson-Hodge 1999).

The second step in this process was estimation of an initial $20-50 \mathrm{keV}$ pulse profile for each segment of data. In each segment, the combined rates were fitted with a model consisting of a sixth-order Fourier expansion in pulse phase (representing the 20-50 keV pulse profile) $r(t)=$ $\sum_{h=1}^{6} \alpha_{h k} \exp \left(i 2 \pi h \phi_{0}(t)\right)$ where $\alpha_{h k}$ is the estimated complex Fourier coefficient for harmonic ${ }^{4} h$ in segment $k$ and a spline function with quadratics

\footnotetext{
${ }^{4}$ In this paper, harmonics are defined as $n \nu$ where $n=$ $1,2,3, \ldots$ and $\nu$ is the pulse frequency.
}

in time (representing the background plus mean source count rate). A sixth-order Fourier expansion was chosen based on the number of harmonics that are significant in a 1-day observation, the typical integration time required to detect XTE J1946+274. Our initial pulse phase model was of the form $\phi_{0}(t)=\nu_{0}\left(t-t_{0}\right)$, where $\nu_{0}=63.1513 \mathrm{mHz}$ before MJD 51270 and $\nu_{0}=$ $63.2800 \mathrm{mHz}$ after MJD 51270 were barycentric frequencies and $t$ was the time corrected to the Solar System barycenter ${ }^{5}$ using the JPL DE-200 ephemeris (Standish et al. 1992). The initial pulse frequency was changed after MJD 51270 because continued spin-up of the pulsar moved the observed frequency too far away from the folding frequency. The value and slope of the spline function were required to be continuous across adjacent 300 $\mathrm{s}$ segment boundaries, but not across data gaps.

Ideally we would like to be able to fit a pulse profile to the entire 4-day interval of data for each frequency grid point; however this is computationally very very expensive. Instead we have formulated an equivalent method in which we first fit pulse profiles to short segments of data using a single fixed frequency. Then the profiles are combined and shifted by a frequency offset corresponding to each grid point. Fitting pulse profiles to short data segments has other advantages in addition to improving computational efficiency. (1) If the folding frequency chosen was incorrect, the profile will be smeared out. However, the degree of smearing in pulse phase $\Delta \phi$ is approximately given by $\Delta \phi=\Delta \nu L_{\mathrm{s}} n$, where $\Delta \nu$ is an offset in frequency, $L_{\mathrm{s}}$ is the segment length, and $n$ is the number of Fourier coefficients. For $L_{\mathrm{s}}=300 \mathrm{~s}$, $\Delta \nu=0.17 \mathrm{mHz}$, and $n=6, \Delta \phi=0.31$, which corresponds to a drop in the amplitude of the sixth harmonic by $15 \%$. The amplitude of the first harmonic only drops by about $0.4 \%$. Using a short segment reduces smearing of the pulse profile. (2) Typically 470 pulse profiles from the $300 \mathrm{~s}$ segments are accumulated within each 4-day interval. Since we have a large number of profiles we can estimate sample variances for each Fourier coefficient. Sample variances give us a measure of the noise present in the data and account for aperiodic noise if it is present.

\footnotetext{
${ }^{5}$ In this paper, the words barycentered and barycentric refer to this correction.
} 
The final step in our advanced pulsar search was a grid search in frequency using the set of typically several hundred estimated 20-50 keV pulse profiles from 4-day intervals of data. New XTE J1946+274 pulse frequencies were determined from an initial grid search over 1200 evenly spaced trial barycentric frequencies in the range 62.97-63.32 mHz for MJD 48361-51270 and 63.10$63.45 \mathrm{mHz}$ for MJD 51270-51690. The search range was changed because continued spin-up of the pulsar moved the pulse frequencies outside the original search range. For each frequency offset $\Delta \nu$, a mean pulse profile is computed for each 4-day interval. The mean pulse profile is of the form

$$
\bar{r}(t)=\sum_{h=1}^{n} \bar{\alpha}_{h} \exp (i 2 \pi h \phi(t))
$$

where $\phi(t)=\phi_{0}(t)+\Delta \phi_{k}, \Delta \phi_{k}=\Delta \nu\left(t_{k}-t_{0}\right)$, and $t_{k}$ is the midpoint barycentric time of segment $k$. The mean coefficients $\bar{\alpha}_{h}$ are given by

$$
\bar{\alpha}_{h}=\sum_{k=1}^{M} w_{k} \alpha_{h k} \exp \left(-i 2 \pi h \Delta \phi_{k}\right)
$$

with weights $w_{k}=\sigma_{\alpha_{h k}}^{-2}\left(\sum_{\ell=1}^{M} \sigma_{\alpha_{h \ell}}^{-2}\right)^{-1}$.

The typical statistic used for a search in pulse frequency or pulse period is the $Z_{n}^{2}$ statistic (Buccheri et al. 1983) given by

$$
Z_{n}^{2}=\sum_{h=1}^{n} \frac{\left|\bar{\alpha}_{h}\right|^{2}}{\sigma_{\bar{\alpha}_{h}}^{2}}
$$

where $\sigma_{\bar{\alpha}_{h}}$ is the formal (i.e., Poisson statistical) error on the Fourier coefficient $\bar{\alpha}_{h}$, respectively. If aperiodic noise is present, either due to XTE $\mathrm{J} 1946+274$ or due to other sources in the large BATSE field of view, e.g., Cygnus X-1, the Poisson statistical error is an underestimate because the actual underlying noise level is higher than Poisson noise. This can create a problem with the $Z_{n}^{2}$ statistic, causing it to depend on the noise level. To account for aperiodic noise, we used all of the typically $\sim 470$ pulse profiles from $300 \mathrm{~s}$ segments within each 4-day interval to estimate sample variances for the Fourier coefficients for each harmonic in the mean profile. Each harmonic was treated separately. We then modified the $Z_{n}^{2}$ statistic by replacing the Poisson variances with our computed sample variances to create a new statistic which we will call $Y_{n}$ after Finger et al. (1999) where it is described in detail. Due to the large field-of-view of BATSE, other pulsars were often also present when we were measuring XTE $\mathrm{J} 1946+274$. If we limited our statistic to use the first 3 harmonics where XTE J1946+274 was the brightest, we reduced the chances of contamination of the search results from other pulsars that happen to have harmonics near the higher harmonics of XTE J1946+274. The best-fit frequency for each 4-day interval was then determined using the $Y_{3}$ statistic.

Using a similar method we also generated pulse frequency measurements for RXTE PCA observations during the initial outburst in 1998 September-October and during the last 2 outbursts, 2001 March-July. Barycentered Standard 1 (125 ms, no energy resolution) data were fitted with a model consisting of a constant background plus a 6th-order Fourier expansion in pulse phase model, creating an estimated 2$60 \mathrm{keV}$ pulse profile for each PCA observation. The pulsed phase model consisted of a constant barycentric frequency, $\nu_{0}=63.17466 \mathrm{mHz}$ for the 1998 September-October observations and $\nu_{0}=63.4239 \mathrm{mHz}$ for the 2001 observations, estimated from projections of BATSE measurements. For each set of observations, we searched over a grid of 151 evenly spaced frequencies spanning the range $\nu_{0} \pm 0.17 \mathrm{mHz}$, the same size interval as used for BATSE.

Figures 1a \& c show the barycentered pulse frequency history and the $2-10 \mathrm{keV}$ total flux history, respectively, for XTE J1946+274. In Figure $1 \mathrm{a}$, the trend in the pulse frequencies repeatedly changes from spin-up to spin down and back to spin-up at regular intervals (every 170 days). This repeated pattern is most likely due to the pulsar's orbit. Within each 170 day cycle in the pulse frequencies, there are two outbursts.

\subsection{Orbit Fitting}

Examination of the observed pulse frequency history showed us two things: (1) a strong orbital signature and (2) strong intrinsic torque variations. To attempt to extract orbital parameters, we first fitted the observed pulse frequencies $\nu_{\mathrm{obs}}$ with a model consisting of a global orbit and a 
global polynomial frequency model, i.e.,

$$
\nu_{\mathrm{obs}}=\nu_{\mathrm{emit}}(1-\beta)
$$

where $\nu_{\text {emit }}=\sum_{k=0}^{n} a_{k}\left(t-t_{0}\right) / k$ ! is a model of the emitted frequency. The velocity relative to the observer $\beta=v / c$ is given by

$\beta=\frac{2 \pi a_{\mathrm{X}} \sin i}{P_{\mathrm{orb}} c}\left\{\frac{\left(1-e^{2}\right)^{1 / 2} \cos \omega \cos E-\sin \omega \sin E}{(1-e \cos E)}\right\}$

where $a_{\mathrm{X}} \sin i$ is the projected semi-major axis of the pulsar's orbit; $P_{\text {orb }}$ is the orbital period; $\omega$ is the periapse angle; and $e$ is the orbital eccentricity; $E$ is the eccentric anomaly, given by

$$
E-e \sin E=2 \pi \frac{\left(t-\tau_{\mathrm{p}}\right)}{P_{\text {orb }}}
$$

and $\tau_{\mathrm{p}}$ is the epoch of periastron passage. We fitted models containing polynomials of orders 1-10. All of these models were very poor fits. Table 2 lists the orbital parameters obtained using a first and 10th order polynomial for comparison. Errors on these parameters have been inflated by a factor of $\left(\chi^{2} / \text { d.o.f. }\right)^{1 / 2}$, but are still expected to be considerably underestimated because the $\chi^{2}$ values are so large. Figure 2 shows the emitted frequency model $\nu_{\text {emit }}, a_{0}(1-\beta)$ the constant term from the emitted frequency model times the velocity relative to the observer to illustrate the amplitude of orbital effects, the full frequency model including the emitted frequency and orbit, and the frequency residuals for the 10th order polynomial model.

Clearly a simple model such as a global polynomial did not suffice to describe the intrinsic torques. Instead we decided to model the intrinsic torques in a piecewise fashion. Each outburst was split into 2-6 intervals, depending on the length of the outburst, where each interval contained 3-4 frequency measurements and spanned 16-24 days or 16-32 days for the BATSE and the RXTE PCA data, respectively. In each interval, we fitted the observed pulse frequencies with a global orbit plus an independent linear frequency model, Equation 4 with $\nu_{\text {emit }}=\nu_{i}+\dot{\nu}_{i}\left(t-t_{i}\right)$ as a model of the emitted frequency for each time interval $i$. Table 2 lists our best fit orbit. Figure 3 shows $\nu_{\text {emit }}$, $\nu_{16}(1-\beta)$ to illustrate the amplitude of orbital variations (Note $\nu_{16}$ was chosen because it lies in near the center of the frequency range.), the full frequency model including the emitted frequency and orbit, and the frequency fit residuals. This fit is not formally acceptable either; however, we believe this is mostly because this model still does not completely describe the intrinsic torques. Errors on the individual parameters in Table 2 have been inflated by $\left(\chi^{2} / \text { d.o.f. }\right)^{1 / 2}$. Comparing the three columns of Table 2 shows that the orbital parameters appear to be similar, whether we use a simple polynomial model or a more complicated piecewise model for $\nu_{\text {emit }}$. Figure $1 b$ shows the orbit-corrected spin frequencies. The regular pattern seen in Figure 1a has been removed, while intrinsic torque effects remain in the data.

\subsection{Orbital Phasing of Outbursts}

A close look at Figure 1 indicates that the outbursts are not fixed in orbital phase and that we are seeing approximately 2 outbursts per orbit. To study the orbital phase of the outbursts, we fitted the 2-10 keV RXTE ASM flux in Figure 1c with a quartic polynomial, and used this polynomial to normalize the outbursts. We then computed the orbital position using

$$
\begin{array}{r}
x=a(\cos E-e) \\
y=a\left(1-e^{2}\right)^{1 / 2} \sin E
\end{array}
$$

where $a$ is the semi-major axis, estimated from $a_{\mathrm{X}} \sin i$ assuming $i=70^{\circ}$ which gives a companion mass of $\sim 14.5 M_{\odot}$. Figure 4 shows the normalized intensity versus orbital position, with darker colors indicating higher intensities. The outer loop of the spiral has $a=323 R_{\odot}$, the estimated radius assuming $i=70^{\circ}$. For each successive orbit, we reduced $a$ by $10 \%$ to offset the orbits. This plot spirals in solely to allow comparison of outburst phases. We are not claiming that the neutron star is spiraling in toward its companion.

The outburst peaks clearly are not fixed in orbital phase, although there is typically one peak on each side of the orbit. Just before periastron and just before apastron, the flux is typically relatively low. The cause of these low flux periods is unclear. Although the fluxes show considerable modulation, they remain detectable with $R X T E$ throughout the period 1998 September - 2001 July. 


\subsection{Spectral Analysis}

To look for variations in energy spectra during the outbursts observed with the RXTE PCA, we first studied hardness ratios. Using FTOOLS ${ }^{6} 5.1$ (Blackburn 1995) and Standard2 (16-s, 129 energy channel) data, we created light curves in 4 energy bands corresponding to 2-5, 5-10, 10-15, and 15-20 keV for both the 1998 and 2001 outbursts. These light curves were background subtracted and their times were corrected to the Solar System barycenter using FTOOLS. Next we formed 3 hardness ratios from adjacent energy bands. Figure 5 shows each of these ratios vs. total PCA source count rate in the $2-30 \mathrm{keV}$ band. Figure 6 shows color-color diagrams. In both figures, the grey-scale denotes intensity, with darker points denoting higher intensities. (Note: This is the measured 2-30 keV intensity, not the normalized 2-10 $\mathrm{keV}$ intensity used in Section 2.3 and Figure 4.) For all of the hardness ratios, we see a correlation between hardness and intensity in the 2001 outburst that is not present in the 1998 outburst. In the color-color diagrams, the hardness ratios are correlated for both sets of outbursts and have similar slopes. However, during the 2001 outbursts, points move from left to right along the correlation as intensity increases; while in the 1998 outburst, the points move around in hardness as intensity increases.

To better quantify the observed spectral changes, we generated response matrices, count spectra, and background spectra using FTOOLS for PCA Standard2 data and HEXTE event mode data for each observation. We then fitted each of the observations in $\mathrm{XSPEC}^{7} 11.1$ (Arnaud 1996) with two models: (1) an absorbed power law with a high-energy cutoff and an iron line, XSPEC model: PHABS (POWERLAW + GAUSSIAN) HIGHECUT $^{7}$ and (2) an absorbed power law with an iron line and a cyclotron absorption feature, XSPEC model: PHABS (POWERLAW + GAUSSIAN) CYCLABS ${ }^{7}$. For both models we used only data in the 2.7-30 keV energy range for PCA data and in the 15-50 keV range for HEXTE. The power law normalizations were allowed to be independent for the PCA and each HEXTE cluster. Figure 7 shows $\chi^{2}$, spectral index, cutoff

\footnotetext{
${ }^{6}$ http://heasarc.gsfc.nasa.gov/ftools

${ }^{7}$ http://xspec.gsfc.nasa.gov
}

energy, folding energy, and flux in the Fe line vs. $2-60 \mathrm{keV}$ flux. Figure 8 shows $\chi^{2}$, spectral index, the cyclotron line energy, and the flux in the $\mathrm{Fe}$ line vs. $2-60 \mathrm{keV}$ flux. Other spectral parameters did not show significant variations with intensity.

A comparison of the two models showed that model 2 generally provided a better fit to the data (see Figures $7 \& 8$, top panel). In both models, the power law photon index remains relatively independent of flux for fluxes above about $10^{-9}$ $\operatorname{ergs~} \mathrm{cm}^{-2} \mathrm{~s}^{-1}$, however, below that flux level, the power law index softens as the intensity decreases. The integrated flux in the iron line is correlated with the 2-60 keV flux in both models, indicating that the iron line is indeed a feature of XTE J1946+274's spectrum and not a background feature. In model 2 a cyclotron absorption feature, when it is constrained, tends to be near the value observed by Heindl et al. (2002).

To estimate an upper limit to the quiescent flux from XTE J1946+274, we fitted PCA data from each of 6 observations after pulsations were undetectable in 2001 August - September with an absorbed power law. These observations were consistent with a power law index of $\sim 2.4$, a hydrogen column of about $6 \times 10^{22} \mathrm{~cm}^{-2}$, and a $2-30 \mathrm{keV}$ flux of $(4-7) \times 10^{-12} \mathrm{ergs} \mathrm{cm}^{-2} \mathrm{~s}^{-1}$. Since RXTE is not an imaging instrument, we cannot distinguish between low-level emission from XTE J1946+274 and background sources such as the galactic ridge. However, this gives us an upper limit on the flux from XTE J1946+274.

\subsection{Spin-up Torque-Flux correlations}

To estimate a bolometric correction to the $R X T E$ ASM measurements, we plotted the 2-60 $\mathrm{keV}$ fluxes from model 2 in Section 2.4 vs. the 5 -day average ASM count rate surrounding each PCA observation. We then fitted a line to these data, obtaining a bolometric correction of 1 ASM ct $\mathrm{s}^{-1}=(1.2 \pm 0.1) \times 10^{-9}$ ergs $\mathrm{cm}^{-2} \mathrm{~s}^{-1}(2-60$ $\mathrm{keV})$. The error on this correction has been estimated including systematic effects. Figure 9 shows the best fit line to these data. Near the peak flux level, the ASM fluxes show evidence for a turnover relative to the PCA fluxes. We have attempted to account for this turn-over by including a systematic error of $10 \%$ on our bolometric correction. Using this bolometric correction, we then plotted the average bolometrically corrected 
ASM flux vs. spin-up rate for each interval in our frequency model. Figure 10 clearly shows a correlation between spin-up rate and flux. Such a correlation suggests a disk is present because direct wind accretion is believed to be less efficient at transferring angular momentum (Ruffert 1997; Benensohn, Lamb, \& Taam 1997; Ruffert 1999). If enough angular momentum is present, a disk will form.

Simple accretion theory assumes that the material from the companion star is flowing onto a rotating neutron star with a strong magnetic field. This field determines the motion of material in a region of space called the magnetosphere. The size of this region is denoted by the magnetospheric radius $r_{m}$, given by (Pringle \& Rees 1972; Lamb, Pethick, \& Pines 1973)

$$
r_{\mathrm{m}} \simeq k\left(G M_{\mathrm{X}}\right)^{-1 / 7} \mu^{4 / 7} \dot{M}^{-2 / 7}
$$

where $G$ is the gravitational constant; $M_{\mathrm{X}}$ is the mass of the neutron star; and $\dot{M}$ is the mass accretion rate, which is assumed to be related to the observed bolometric flux $F$ by $\dot{M}=4 \pi d^{2} F R_{\mathrm{X}} / G M_{\mathrm{X}}$, where $R_{\mathrm{X}}$ is the radius of the neutron star and $d$ is the distance to the pulsar. $k$ is a constant factor of order 1 . Equation 9 with $k \simeq 0.91$ gives the Alfvén radius $r_{A}$ for spherical accretion and with $k \simeq 0.47 n\left(\omega_{s}\right)$ gives the magnetospheric radius derived by Ghosh \& Lamb (1979).

The torque applied by accretion of matter onto a neutron star, assuming torques due to matter leaving the system are negligible, is given by (Lamb, Pethick, \& Pines 1973)

$$
\frac{d}{d t}(2 \pi I \nu)=\dot{M} \ell
$$

where $I$ is the moment of inertia of the neutron star and $\ell$ is the specific angular momentum of the material. If $I$ is assumed constant, then $\ell$ is given by

$$
\ell=2 \pi I \dot{\nu} \dot{M}^{-1},
$$

where $\dot{\nu}$ is the spin-up rate. To estimate $\ell$ for XTE J1946+274, we assumed typical pulsar parameters, $M_{\mathrm{X}}=1.4 M_{\odot}, R_{\mathrm{X}}=10 \mathrm{~km}, I=10^{45} \mathrm{~g}$ $\mathrm{cm}^{2}$, typical values of $\dot{\nu} \simeq 5 \times 10^{-12} \mathrm{~Hz} \mathrm{~s}^{-1}$ and $F_{\text {bol }} \simeq 2 \times 10^{-9} \mathrm{ergs} \mathrm{cm}^{-2} \mathrm{~s}^{-1}$ (see Figure 10), and a distance $d=8-10 \mathrm{kpc}$ (Verrecchia et al. 2002). This yielded $\ell=(2.4-3.8) \times 10^{17} \mathrm{~cm}^{2} \mathrm{~s}^{-1}$.
An accretion disk will form if the specific angular momentum of the material accreted from the Be star's disk is comparable to the Keplerian specific angular momentum at the magnetospheric radius, i.e.,

$$
\ell \simeq \ell_{\mathrm{m}}=\left(G M_{\mathrm{X}} r_{m}\right)^{1 / 2} .
$$

For XTE J1946+274, using the magnetic field measurement $B=3.1(1+z) \times 10^{12} \mathrm{G}$ (Heindl et al. 2002) which implies $\mu=1.6 \times 10^{30} \mathrm{G} \mathrm{cm}^{3}$, then $\ell_{\mathrm{m}}=(2.7-2.8) \times 10^{17} \mathrm{~cm}^{2} \mathrm{~s}^{-1}$ for $k=0.91$ and $\ell_{\mathrm{m}}=(1.9-2.0) \times 10^{17} \mathrm{~cm}^{2} \mathrm{~s}^{-1}$ for $k=0.47$. For XTE J1946+274, $\ell=0.9-1.3 \ell_{\mathrm{m}}$ for $k=0.91$ and $\ell=1.3-1.9 \ell_{\mathrm{m}}$ for $\mathrm{k}=0.47$, hence an accretion disk is most likely present. In contrast, for the wind-fed system Vela X-1 where a disk is not expected to be present, $\dot{\nu} \simeq 6 \times 10^{-14} \mathrm{~Hz}$ $\mathrm{s}^{-1}$ (Inam \& Baykal 2000), $L \simeq 2 \times 10^{38} \mathrm{ergs}$ $\mathrm{s}^{-1}$, and $\mu \simeq 2.1 \times 10^{30} \mathrm{G} \mathrm{cm}^{3}$ (Makishima et al. 1999) leading to $\ell \simeq 3.5 \times 10^{14} \mathrm{~cm}^{2} \mathrm{~s}^{-1}$ and $\ell_{\mathrm{m}} \simeq 2.2 \times 10^{17} \mathrm{~cm}^{2} \mathrm{~s}^{-1}$, i.e., $\ell \simeq 0.002 \ell_{\mathrm{m}}$. Further, three-dimensional simulations of wind accretion show that the average specific angular momentum accreted via wind accretion is always smaller than the Keplerian value (Ruffert 1997, 1999).

Since an accretion disk appears to be present, we can use simple accretion theory to derive a distance to XTE J1946+274. Substituting Equations 12 into Equation 10 and solving for $\dot{\nu}$ gives

$$
\dot{\nu}=1.39 k^{1 / 2}\left(G M_{\mathrm{X}}\right)^{-3 / 7} R_{\mathrm{X}}^{6 / 7} I^{-1} \mu^{2 / 7} d^{12 / 7} F^{6 / 7} .
$$

Assuming $k=0.91$ and the parameters defined earlier,

$$
\dot{\nu}_{12}=5.8 \times 10^{-2} d_{\mathrm{kpc}}^{12 / 7} F_{9}^{6 / 7},
$$

where $\dot{\nu}_{12}$ is the spin-up rate in units of $10^{-12} \mathrm{~Hz}$ $\mathrm{s}^{-1}$ and $F_{9}$ is the bolometric flux in units of $10^{-9}$ ergs $\mathrm{cm}^{-2} \mathrm{~s}^{-1}$. We then fitted this model to the data in Figure 10. To account for the flux and $\dot{\nu}$ errors, we computed $\chi^{2}$ as

$$
\chi^{2}=\sum_{i=1}^{n}\left\{\frac{\left(F_{i}-F_{i}^{\text {mod }}\right)^{2}}{\sigma_{F_{i}}^{2}}+\frac{\left(\dot{\nu}_{i}-\dot{\nu}^{\bmod }\left(F_{i}^{\text {mod }}\right)\right)^{2}}{\sigma_{\dot{\nu}_{i}}^{2}}\right\}
$$

where $F_{i}^{\text {mod }}$ is a free parameter fitted to each flux and $\dot{\nu}^{\bmod }\left(F_{i}^{\bmod }\right)$ is Equation 14 evaluated at $F_{i}^{\text {mod }}$. The solid line shows our best fit, with 
$\chi^{2} / 32=1.0$, which gives us a distance of $9.5 \pm 0.3$ kpc. This distance is consistent with optical observations of the counterpart. The errors on the distance are computed from the fit itself and take into account only the statistical errors on the flux and spin-up rate and the estimated systematic error on the bolometric correction. However, there is considerable uncertainty in our assumed neutron star parameters. The estimated distance depends on the following combination of neutron star parameters: $M_{x}^{1 / 4} R_{x}^{-1 / 2} I^{7 / 12}$. Using the neutron star equations of state given by Wiringa, Fiks, \& Fabriocini (1988) and assuming $M_{x}=1.0-1.8 M_{\odot}$, we estimate the error on the distance due to uncertainty in the neutron star parameters is about $30 \%$, i.e., $d=9.5 \pm 2.9 \mathrm{kpc}$.

\subsection{Pulse Profiles}

Using FTOOLS and RXTE PCA event mode data, we created light curves and corresponding background light curves in 5 energy bands: 2-5, $5-10,10-15,15-20$, and $20-30 \mathrm{keV}$ for each of 29 observations, 12 from the 1998 outburst and 17 from the 2001 outbursts. Each light curve was barycentered, background subtracted, and folded at the appropriate frequency from our frequency search. Each pulse profile contained 50 phase bins. First we examined profiles from the entire 2-30 $\mathrm{keV}$ band to search for intensity dependent variations. We aligned all of the pulse profiles by finding the phase of the minimum of the profile using a quadratic interpolation and placing that minimum at phase 0.0. We found definite variations with intensity in both the 1998 and 2001 outbursts and also evidence that the pulse profile was different at similar intensities during the rise and fall of the initial bright outburst.

Profiles with similar shapes and similar intensities were averaged to better illustrate the observed shape changes. Figure 11a shows the average 2-30 $\mathrm{keV}$ pulse profile at 6 different intensities. Figure $11 \mathrm{~b}$ shows the peak-to-peak pulse fraction versus mean flux for each profile. In the following descriptions and in Figures 11a and b, the pulse profiles are numbered from one to six. (1) At the lowest intensities (6-17 counts s ${ }^{-1} \mathrm{PCU}^{-1}, 2-30 \mathrm{keV}$ ), during the 2001 outbursts, the profile's main feature was a deep notch, which we used to align the profiles. The profile consisted of an asymmetric structured main peak that peaked near phase 0.2 .
(2) As the intensity increased (23-65 counts $\mathrm{s}^{-1}$ $\mathrm{PCU}^{-1}$ ), a second notch becomes prominent near phase 0.45 , making the profile consist of two main peaks. These peaks were approximately equal in intensity at first, but the second peak brightens as the overall intensity increases. A small peak near phase 0.87 also appears. No RXTE PCA observations were taken at intensities between 65 and 178 counts s ${ }^{-1} \mathrm{PCU}^{-1}$. (3) The next profile shape occurs at intensities of $178-266$ counts s ${ }^{-1} \mathrm{PCU}^{-1}$, near the end of the 1998 outburst. This profile is markedly different from that near the peak of the 2001 outburst (profile 2). Both notches are broader, the peak near phase 0.65 is brighter than the peak near phase 0.3 and the small peak at phase 0.87 has disappeared. The main notch appears to be less deep than at lower intensities. (4) Profiles at intensities of 285-308 and 330 counts $\mathrm{s}^{-1} \mathrm{PCU}^{-1}$, all from the rise of the 1998 outburst, show a broader first peak and a less intense shoulder following the second peak than the lower intensity profiles from the fall of the outburst (profile 3 ). (5) At intensities of $322-328$ counts s ${ }^{-1} \mathrm{PCU}^{-1}$, during the decline of the 1998 outburst, the first peak is narrower than at similar intensities during the outburst rise (profile 4), but broader than at lower intensities during the decline (profile 3 ). (6) At the peak of the outburst, the width of the first peak is at an intermediate width between the profiles from the rise and fall of the outburst. During the 2001 outbursts, the profile shape appeared to depend primarily on intensity, while during the 1998 bright outburst, the profile shape depended on both intensity and whether the profile was from the rise or fall of the outburst.

Next we looked for variations in the pulse profile versus energy. Figure 12 shows pulse profiles in 5 energy bands from the peak of the 1998 outburst (left panel) and near one of the peaks in the pair of outbursts in 2001 (right panel). In the 1998 profiles, the profile consists of two main peaks at lower energies $(\lesssim 15 \mathrm{keV})$. The first peak is dominant at lower energies and the second peak becomes more dominant as energy increases. Also, as energy increases, the notch between the two main peaks fills in. As the 2-30 keV intensity decreases, the profile "shape" appears to move down in energy. For example, in the right hand panel of Figure 12, the 2-5 keV profile consists of two nearly equal peaks, reminiscent of the $10-15 \mathrm{keV}$ profile at the peak of 
the 1998 outburst.

\subsection{Optical Observations}

A regular monitoring program was established to study the strength and structure of the $\mathrm{H} \alpha$ optical emission line during the X-ray observations in 2001. This line arises from the circumstellar disk surrounding the Be star and the line properties are strongly related to the extent and dynamic structure of the disk. Data were primarily collected from the $2.5 \mathrm{~m}$ Isaac Newton Telescope (INT) in La Palma, Spain. The INT was equipped with the 235-mm camera and EEV\#10 CCD. The use of the R1200R grating results in a nominal dispersion of $\approx 0.40 \AA /$ pixel. Intermediate resolution spectroscopy was also performed on 17th July 2001 and 22nd October 2001 using the 1.93-m telescope at the Observatoire de Haute Provence, France. That telescope was equipped with the long-slit spectrograph Carelec and the $1024 \times 2048$ EEV CCD. We used the $1200 \mathrm{ln} / \mathrm{mm}$ grating in first order, resulting in a nominal dispersion of $\approx 0.45 \AA /$ pixel. See Table 3 for details.

The $\mathrm{H} \alpha$ profile obtained from each observation is presented in Figure 13. The lines have been shifted vertically by an arbitrary amount in order to present each profile clearly. The data of the 17 July and 7 October 2001 are of a lower standard due to poor weather conditions. Nonetheless it is possible to accurately determine the equivalent width of each of the emission lines; values are presented in Table 3. From these numbers one can see that there is little evidence for any changes in the line flux, assuming a constant continuum. However, from Figure 13, one can see obvious shifts in the line position after 2001 July.

If one takes the first spectrum (3 May 2001) as a base line and subtracts it from each of the other spectra after they have been normalized to the same peak value, then significant changes are apparent. The result of this process is shown in Figure 14. It is apparent that little happens to the profiles (and, presumably, the circumstellar disk) until July/August 2001. Starting with the spectrum of 17 July 2001 one can see significant perturbations occurring in the line profile indicative of density changes in the disk structure. Between August and September the direction of the perturbation changes, suggesting that the density enhancement/rarefication had changed sides (or ro- tated around) in the disk. The size of the perturbation also seems to have been increasing from July to October, though the separation of the red and blue peaks remains approximately constant at $\sim 340 \mathrm{~km} / \mathrm{s}$.

\section{Discussion}

XTE J1946+274 is a very unusual Be/X-ray binary system and its behavior does not fit well into the standard normal/giant outburst behavior. Instead it showed an extended period of activity from 1998 September - 2001 July when the $\mathrm{X}$-ray flux showed considerable modulation, with two peaks or outbursts per orbit, but the X-ray flux never dropped below the detection threshold of $R X T E$. This extended period of activity more closely resembles a series of normal outbursts than a single giant outburst; however, unlike typical normal outbursts, these outbursts shift rapidly in orbital phase, there are two outbursts per orbit, and the X-ray flux does not drop dramatically between outbursts. The initial outburst shows considerable spin-up $\dot{\nu} \gtrsim 10^{-11} \mathrm{~Hz} \mathrm{~s}^{-1}$, like a giant outburst, but this spin-up is not substantially larger than that seen in later outbursts. In addition, the peak flux $(2-60 \mathrm{keV})$ of the initial outburst is about $7 \times 10^{-9} \mathrm{ergs} \mathrm{cm}^{-2} \mathrm{~s}^{-1}$, while the peak flux $(2-60 \mathrm{keV})$ of the last two outbursts observed with the PCA is about $2 \times 10^{-9}$ ergs $\mathrm{cm}^{-2} \mathrm{~s}^{-1}$, only a factor of $\sim 3.5$ fainter than the brightest outburst. These fluxes correspond to luminosities of $8 \times 10^{37} \mathrm{ergs} \mathrm{s}^{-1}$ and $2 \times 10^{37} \mathrm{ergs}$ $\mathrm{s}^{-1}$, respectively, assuming our best-fit distance of $9.5 \mathrm{kpc}$.

In giant outbursts of $\mathrm{Be} / \mathrm{X}$-ray binaries, accretion disks are expected to be present and indeed, evidence for an accretion disk, based on correlations between the observed flux and spin-up rate, has been found for several sources during giant outbursts (Parmar et al. 1989; Reynolds et al. 1996; Bildsten et al. 1997; Wilson et al. 1998; Stollberg et al. 1999). Independent evidence for an accretion disk based on the detection of quasiperiodic oscillations during a giant outburst has been found for EXO 2030+375 (Angelini, Stella, \& Parmar 1989) and A0535+262 (Finger, Wilson, \& Harmon 1996). Until recently, normal outbursts were believed to be due to direct wind accretion from the Be disk, so significant spin-up was not 
expected because direct wind accretion is not believed to be very efficient at transferring angular momentum (Ruffert 1997; Benensohn, Lamb, \& Taam 1997; Ruffert 1999). If enough angular momentum is present in the accreted material, an accretion disk will form. However, evidence for spinup during normal outbursts has been observed in GS 0834-430 (Wilson et al. 1997), 2S 1417-624 (Finger, Wilson, \& Chakrabarty 1996), 2S 1845024 (Finger et al. 1999), and in EXO 2030+375 (Stollberg et al. 1999; Wilson et al. 2002).

We see a correlation between spin-up and flux for XTE J1946+274 as shown in Figure 10. The spin-up rate and its correlation with bolometric flux during XTE J1946+274's outbursts suggest an accretion disk may be present. In addition, our calculations show that the specific angular momentum of the accreted material is comparable to the Keplerian specific angular momentum, hence a disk is expected to form in XTE J1946+274. Further, our fit to the spin-up vs. bolometric flux correlation, which assumed a disk was present, yielded a distance of $9.5 \pm 2.9 \mathrm{kpc}$ which is consistent with the distance of 8-10 kpc derived from optical observations Verrecchia et al. (2002). Hence an accretion disk is likely to be present in XTE J1946+274.

Between 2001 July 31 (MJD 52121) and 2001 August 9 (MJD 52130), XTE J1946+274 dropped below the PCA's detection threshold. At approximately the same time, between 2001 June 29 (MJD 52089) and 2001 July 17 (MJD 52107), the $\mathrm{H} \alpha$ profile began to change rapidly. The profile was stable for 3 observations on 2001 May 3, May 10, and June 29 (MJD 52032, 52039, \& 52089) that corresponded to X-ray observations during the decline of the second to last outburst, during the low state between the last two outbursts, and near the peak of the last outburst. The coincidence of the change in $\mathrm{H} \alpha$ with the X-ray turn-off suggests that changes in the Be disk caused the X-ray outbursts to cease.

To determine whether or not the observed Xray turn-off was due to centrifugal inhibition of accretion (Stella, White, \& Rosner 1986), we estimate the flux at the onset of this effect by equating the magnetospheric radius to the corotation radius. The magnetospheric radius is given by
Equation 9 and the corotation radius is given by

$$
r_{\mathrm{co}}=(G M)^{1 / 3}(2 \pi \nu)^{-2 / 3}
$$

where $\nu$ is the spin frequency of the pulsar. Setting $r_{\mathrm{m}}=r_{\text {co }}$ gives the threshold flux for the onset of centrifugal inhibition of accretion, i.e.,

$F_{\mathrm{x}}^{\mathrm{min}} \simeq 3.0 \times 10^{-9} \mathrm{ergs} \mathrm{cm}^{-2} \mathrm{~s}^{-1} k^{7 / 2} \mu_{30}^{2} M_{1.4}^{-2 / 3} R_{6}^{-1} P_{15.8 \mathrm{~s}}^{-7 / 3} d_{\mathrm{kpc}}^{-2}$

where $\mu_{30}, M_{1.4}, R_{6}$, and $P_{15.8 \mathrm{~s}}$ are the pulsar's magnetic moment in units of $10^{30} \mathrm{G} \mathrm{cm}^{3}$, mass in units of $1.4 M_{\odot}$, radius in units of $10^{6} \mathrm{~cm}$, and spin period in units of 15.8 seconds, respectively. Using $d=9.5 \mathrm{kpc}$ and $\mu=1.6 \times 10^{30} \mathrm{G} \mathrm{cm}^{3}$ yields $F_{\mathrm{x}}^{\min } \simeq 1.2 \times 10^{-10} \mathrm{ergs} \mathrm{cm}^{-2} \mathrm{~s}^{-1} k^{7 / 2}$. For $k=0.47-0.91, F_{\mathrm{x}}^{\min } \simeq(0.6-6.0) \times 10^{-11} \mathrm{ergs}$ $\mathrm{cm}^{-2} \mathrm{~s}^{-1}$. Our measured upper limit fluxes are in the range $(4-7) \times 10^{-12}$ ergs $\mathrm{cm}^{-2} \mathrm{~s}^{-1}$, consistent with XTE J1946+274 entering the centrifugal inhibition of accretion regime when the X-rays became undetectable with the PCA.

\section{Conclusions}

We propose that XTE J1946+274 is a system in which the Be star's equatorial plane and the orbital plane are not aligned. This is suggested by: (1) The optical observations show a relatively narrow FWHM (8.6 ̊ on 2001 May 10, which corresponds to $\pm 200 \mathrm{~km} \mathrm{~s}^{-1}$ ) for the single-peaked $\mathrm{H} \alpha$ line, indicating that the Be star is viewed from a relatively low inclination angle, i.e., nearly poleon. (2) The orbital signature is quite obvious in the pulse frequencies, indicating that the orbital inclination angle is likely not low: the derived mass function $f(M)=9.7_{-4.5}^{+6.9} M_{\odot}$ from the piecewise frequency model indicates an inclination angle $\gtrsim 46^{\circ}$ for the expected mass range of $10-16 M_{\odot}$ derived from optical observations. Figure 15 shows the range of allowed inclinations using the 1- $\sigma$ errors on the mass function.

We see two outbursts per orbit in XTE J1946+274; however, these outbursts are not fixed in orbital phase. This combination of behaviors is unique to XTE J1946+274 and is likely not due to a single mechanism. The optical data and mass function suggest that the orbital plane and the Be disk are not aligned in this system. Such a misaligned system would be expected to produce two outbursts per orbit, each approximately corresponding to 
the neutron star's passage through the Be disk; hence these outbursts would be expected to be fixed in orbital phase. For XTE J1946+274, we are faced with the difficult task of explaining not only why we see the expected two outbursts per orbit, but also why these outbursts are not fixed in orbital phase. We propose that given the relatively high luminosities and spin-up rates in all of the outbursts that perhaps XTE J1946+274's unusual outburst behavior can be explained as a giant outburst. In normal outbursts, the Be disk is expected to be truncated at a resonance radius by tidal forces from the neutron star's orbit (Okazaki \& Negueruela 2001); however, in giant outbursts, where much more material is believed to be present in the disk, it is believed that the disk is no longer truncated. Hence more material likely means a larger disk around the Be star. Density perturbations propagating in the Be disk could change the contact points of the disk and the orbit. These perturbations would have to be moving quite rapidly in the disk. This idea of a giant outburst combined with density perturbations seems most likely to work in a system where the inclination angle between the Be disk and the orbital plane is fairly small. It is not clear that this angle is small in XTE J1946+274. We do not claim that the idea of a giant outburst in an inclined system fully explains the behavior observed from XTE J1946+274. Instead we put the idea forth as a suggestion to those doing simulations of these systems.

The phasing of the outbursts of XTE J1946+274 (see Figure 4) is difficult to understand. However, it is not the first Be/X-ray binary to show shifts in outburst phase. EXO 2030+375 underwent a fairly sudden (within 4 orbits) shift in the peak phase of the outbursts from 6 days after periastron (phase $\sim 0.13$ ) to 2.5 days before periastron (phase -0.05), followed by a gradual shift in outburst phase to 2.5 days after periastron (phase 0.05). This shift was believed to be associated with a density perturbation observed in the $\mathrm{Be}$ disk via $\mathrm{H} \alpha$ observations (Wilson et al. 2002). GS 0834-430 also underwent sudden shifts in outburst phase, undergoing 9 outbursts approximately centered on periastron, followed by dramatic shifts to outbursts centered on phases 0.37 and 0.75 (Wilson et al. 1997). A companion was not known at the time for GS $0834-430$, so we cannot confirm that density perturbations in the Be disk were responsible for these phase shifts. In XTE J1946+274, we have a similar problem, with the companion not observed for much of the time the X-ray outbursts were occurring. In addition, the problem is compounded by the fact that we are likely viewing the Be star nearly pole-on; hence the range of projected rotational velocities is very small, making it difficult to detect perturbed velocities which indicate density perturbations. Only large density perturbations, such as the one that coincided with the X-ray turn-off, are likely to be detected.

We are grateful to Luisa Morales for providing one of the optical spectra. This research has made use of data obtained from the High Energy Astrophysics Science Archive Research Center (HEASARC), provided by NASA's Goddard Space Flight Center (GSFC). RXTE ASM quicklook results were provided by the ASM/RXTE teams at MIT and at the GSFC SOF and GOF. The INT is operated on the island of La Palma by the Isaac Newton Group in the Spanish Observatorio del Roque de los Muchachos of the Instituto de Astrofísica de Canarias. Based in part on observations made at Observatoire de Haute Provence (CNRS), France. 


\section{REFERENCES}

Angelini, L., Stella, L., \& Parmar, A.N. 1989, ApJ, 346,906

Arnaud, K.A. 1996, Astronomical Data Analysis Software and Systems V, eds. G. Jacoby \& J. Barnes, ASP Conf. Series, 101, 17

Benensohn, J.S., Lamb, D.Q. \& Taam, R.E. 1997, ApJ, 478, 723

Bildsten, L. et al. 1997, ApJS, 113, 367

Blackburn, J.K. 1995, in ASP Conf. Ser., Vol. 77, Astronomical Data Analysis and Software Systems IV, ed. R.A. Shaw, H.E. Payne, \& J.J.E. Haynes (San Francisco:ASP), 367

Buccheri, R. et al. 1983, A\&A, 128, 245

Campana, S., Israel, G., \& Stella, L. 1998, IAU Circ. 7039

Campana, S., Israel, G., \& Stella, L. 1999, A\&A, 352, L91

Coe, M.J. 2000, in The Be Phenomenon in EarlyType Stars, IAU Colloquium 175, ASP Conf. Proc. 214, eds. M.A. Smith \& H.F. Henrichs, (San Francisco:ASP), 656

Corbet, R.H.D. 1986, MNRAS, 220, 1047

Finger, M. H. et al. 1999, ApJ, 517, 449

Finger, M.H., Wilson, R.B. \& Chakrabarty, D. 1996, A\&AS, 120, 209

Finger, M.H., Wilson, R.B., Harmon, B.A. 1996, ApJ, 459, 288

Fishman, G. J. et al. 1989, in Proc. GRO Science Workshop, ed. W.N. Johnson (Greenbelt: NASA/GSFC), 2

Ghavamian, P. \& Garcia, M. 1998, IAU Circ. 7022

Ghosh, P. \& Lamb, F.K. 1979, ApJ, 234, 296

Hanuschik, R.W. 1996, A\&A, 308, 170

Heindl, W.A. et al. 2002, ApJ, 563, L35

Inam, S.C. \& Baykal, A. 2000, A\&A, 353, 617

Israel, G.L., Polcaro, V.F., \& Covino, S. 1998, IAU Circ. 7021
Jahoda, K. et al. 1996, EUV, X-ray, and GammaRay Instrumentation for Astronomy VII, SPIE Proc. 2808, ed. O.H.V. Sigmund \& M.Gumm (Bellingham:SPIE), 59

Lamb, F.K., Pethick, C.J., \& Pines, D. 1973, ApJ, 184,271

Levine, A.M., Bradt, H., Cui, W., Jernigan, J.G., Morgan, E.H., Remillard, R., Shirey, R. E., \& Smith, D.A. 1996, ApJ, 469, L33

Makishima, K., Mihara, T., Nagase, F., Tanaka, Y. 1999, ApJ, 525, 978

Okazaki, A.T. \& Negueruela, I. 2001, A\&A, 377, 161

Parmar, A.N., White, N.E., Stella, L., \& Ferri, P. 1989, ApJ, 338, 359

Paul, B. et al. 2001, A\&A, 370, 529

Pringle, J.E., \& Rees, M.J. 1972, A\&A, 21, 1

Quirrenbach, A. et al. 1997, ApJ, 479, 477

Reynolds, A.P. et al. 1996, A\&A, 312, 872

Rothschild, R.E. et al. 1998, ApJ, 496, 538

Ruffert, M. 1997, A\&A, 317, 793

Ruffert, M. 1999, A\&A, 346, 861

Slettebak, A. 1988, PASP, 100, 770

Smith, D.A. \& Takeshima, T. 1998, IAU Circ. 7014

Standish, E. M., Newhall, X. X., Williams, J. G., \& Yeomans, D. K. 1992, Orbital ephemerides of the Sun, Moon, \& Planets, in Explanatory Supplement to the Astronomical Almanac, ed. P. K. Seidelmann (Mill Valley: University Science Books), 279

Stella, L., White, N.E., \& Rosner, R. 1986, ApJ, 308,669

Stollberg, M.T. et al. 1999, ApJ, 512, 313

Takeshima, T. \& Chakrabarty, D. 1998, IAU Circ. 7016

Verrecchia, F. et al. 2002, A\&A, in press 
Waters, L.B.F.M. \& van Kerkwijk, M.H. 1989, A\&A, 223, 196

Wilson, C.A. et al. 1997, ApJ, 479, 388

Wilson, C.A., Finger, M.H., Wilson, R.B., Scott, D.M. 1998, IAU Circ. 7014

Wilson, C.A., Finger, M.H., Coe, M.J., Laycock, S., Fabregat, J. 2002, ApJ, 570, 287

Wilson-Hodge, C.A. 1999, PhD Dissertation, University of Alabama in Huntsville

Wiringa, R.B., Fiks, V. \& Fabriocini, A. 1988, Phys. Rev. C., 38, 1010

This 2-column preprint was prepared with the AAS LATEX macros v5.0.

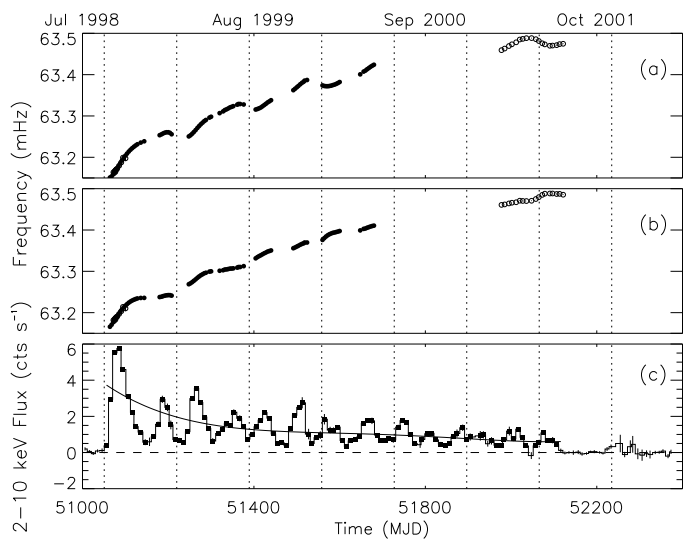

Fig. 1. - XTE J1946+274 outburst history. (a) Barycentered pulse frequency measurements with BATSE (filled circles) and RXTE PCA (open circles). (b) The pulse frequency measurements shown in (a) have been corrected for our orbital solution listed in the fourth column of Table 2 (c) $2-10 \mathrm{keV}$ flux history measured with the $R X T E$ ASM. Each point is a 10-day average flux. Filled squares denote $3-\sigma$ or better detections. The solid curve denotes a quartic polynomial fitted to the $R X T E$ ASM flux (see Section 2.3). Vertical dotted lines in all three panels denote the estimated times of periastron passage using the orbital parameters in the fourth column of Table 2 . 
TABLE 1

Observations of the Sky Region Including XTE J1946+274

\begin{tabular}{cclll}
\hline \hline Dates & \multicolumn{1}{c}{ MJD } & \multicolumn{1}{c}{ Instrument $^{*}$ No. Obs. $^{\text {a }}$} & ${\text { Total PCA Exposure }(\mathrm{ks})^{\mathrm{a}}}$ \\
\hline 1991 Apr 15 - 2000 May 27 & $48361-51691$ & BATSE & $\ldots$ & $\ldots$ \\
1996 Feb 23 - 2002 Apr 11 & $50136-52376$ & $R X T E$ ASM & $\ldots$ & $\ldots$ \\
1998 Sep 16 - 1998 Oct 14 & $51072-51100$ & $R X T E$ PCA, HEXTE & 12 & 85.87 \\
2001 Mar 9 - 2001 Sep 25 & $51977-52177$ & $R X T E$ PCA, HEXTE & 24 & 129.49 \\
\hline
\end{tabular}

${ }^{\mathrm{a}}$ For pointed observations.

TABle 2

Orbit Fit

\begin{tabular}{llll}
\hline \hline \multicolumn{1}{c}{ Parameter } & \multicolumn{1}{c}{ Linear $\nu_{\text {emit }}$} & 10th Order $\nu_{\text {emit }}$ & Piecewise $\nu_{\text {emit }}$ \\
\hline$P_{\text {orb }}($ days $)$ & $173 \pm 2$ & $167.8 \pm 0.6$ & $169.2 \pm 0.9$ \\
$\tau_{\mathrm{p}}$ & JD2451524.6 \pm 16.9 & JD2451572.3 \pm 1.6 & JD2451558.7 \pm 4 \\
$a_{\mathrm{X}} \sin i($ lt-sec $)$ & $972 \pm 118$ & $474 \pm 14$ & $640 \pm 120$ \\
$e$ & $0.19 \pm 0.13$ & $0.35 \pm 0.03$ & $0.33 \pm 0.05$ \\
$\omega($ degrees $)$ & $-153 \pm 37$ & $-38 \pm 5$ & $-91 \pm 23$ \\
$f(M)\left(M_{\odot}\right)$ & $32 \pm 11$ & $4.0 \pm 0.3$ & $9.7_{-4.5}^{+6.9}$ \\
$\chi^{2} /$ d.o.f & $2699556 / 101$ & $28756 / 92$ & $220.1 / 37$ \\
\hline
\end{tabular}

TABLE 3

JOURNAL OF OPTICAL OBSERVATIONS.

\begin{tabular}{cccccc}
\hline \hline Date & Telescope $^{\mathrm{a}}$ & Instrument $^{\mathrm{b}}$ & Grating & Detector & $\mathrm{H} \alpha$ EW $(\AA)$ \\
\hline 3 May 2001 & INT & IDS+500 camera & R1200R & TEK5 & $45.9 \pm 0.6$ \\
10 May 2001 & INT & IDS+235 camera & R1200R & EEV10 & $41.0 \pm 0.8$ \\
29 Jun 2001 & INT & IDS+235 camera & R1200R & EEV10 & $41.1 \pm 0.3$ \\
17 Jul 2001 & OHP & Carelec & $12001 / m m$ & EEV & $39.1 \pm 0.4$ \\
12 Aug 2001 & INT & IDS+235 camera & R1200R & EEV10 & $43.9 \pm 0.3$ \\
26 Sep 2001 & INT & IDS+235 camera & R1200R & EEV10 & $43.6 \pm 0.3$ \\
7 Oct 2001 & INT & IDS+235 camera & R1200R & EEV10 & $38.5 \pm 0.5$ \\
22 Oct 2001 & OHP & Carelec & $12001 / m m$ & EEV & $42.5 \pm 0.3$ \\
\hline
\end{tabular}

${ }^{\mathrm{a}}$ INT $=$ Isaac Newton Telescope, OHP $=$ Observatoire de Haute Provence

${ }^{\mathrm{b}} \mathrm{IDS}=$ Intermediate Dispersion Spectrograph. 


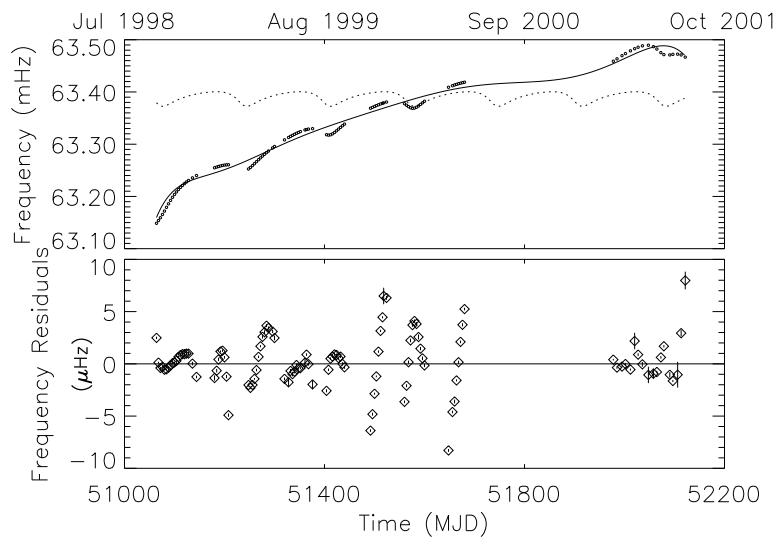

Fig. 2.- Top: The solid curve denotes the emitted frequency modeled as a 10th order polynomial. The dotted curve illustrates the magnitude of orbital effects. The open circles denote the full pulse frequency model including both the 10th order polynomial and the orbital effects. Bottom: Pulse frequency residuals for the model consisting of a global orbit and a global 10th order emitted frequency model.

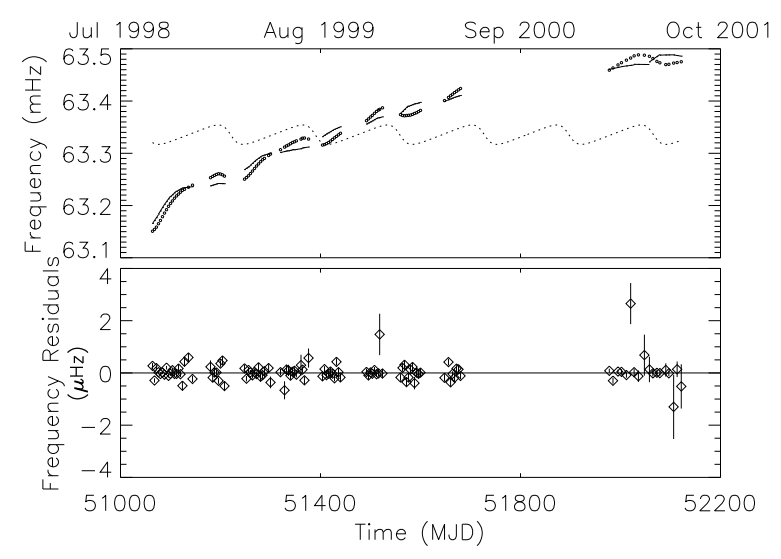

Fig. 3.- Top: The broken curve denotes the piecewise emitted frequency model. The dotted curve illustrates the magnitude of orbital effects. The open circles denote the full pulse frequency model including both the piecewise emitted frequency model and the orbital effects. Bottom: Pulse frequency residuals for the model consisting of a global orbit and piecewise emitted frequency model.

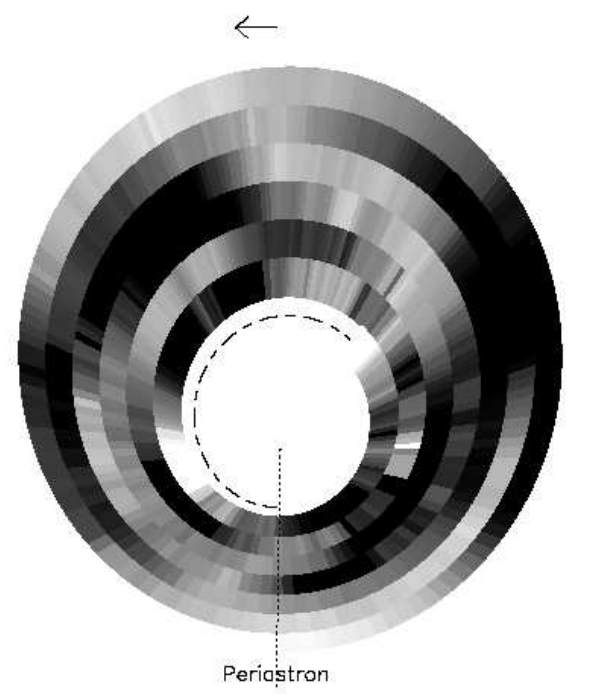

Fig. 4.- Normalized intensity (darker colors indicate higher intensities) vs. position in the orbit. The outside loop is the shape of the actual orbit. The semi-major axis of each successive orbit is reduced by $10 \%$ to offset the orbits, allowing comparison of the orbital phase of the outburst in successive orbits. This plot is not meant to indicate that the neutron star is spiraling in toward its companion.

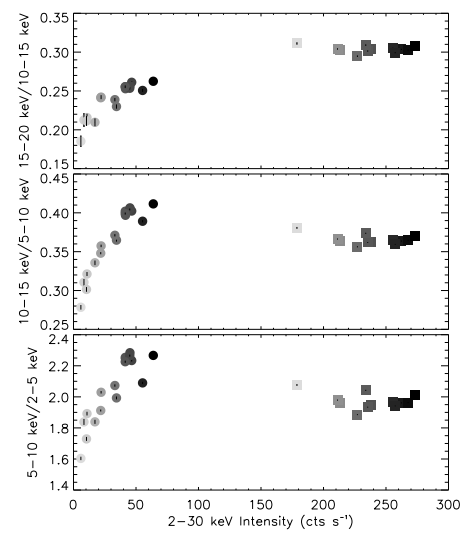

Fig. 5.- Hardness-intensity diagrams. Filled squares denote points from the 1998 outburst and filled circles denote the 2001 outbursts. The color darkens as the intensity increases within each outburst to allow comparison with Figure 6 

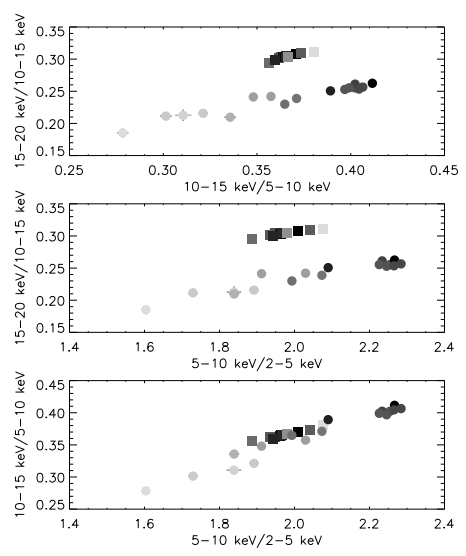

Fig. 6.- Color-color diagrams. Filled squares denote points from the 1998 outburst and filled circles denote the 2001 outbursts. For both sets of outbursts, the color darkens as the intensity increases.

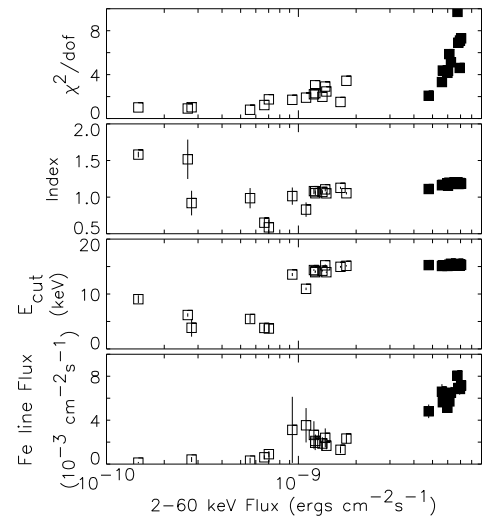

Fig. 7.- Spectral fitting results for an absorbed power law with a high energy cutoff and a Gaussian iron line. Shown are the goodness of fit $\chi^{2} /$ d.o.f., the power law photon index, the cut off energy $E_{\text {cut }}$, and the integrated flux in the iron line versus $2-60 \mathrm{keV}$ flux. Filled squares denote the 1998 outburst and open squares denote the last pair of outbursts observed in 2001.

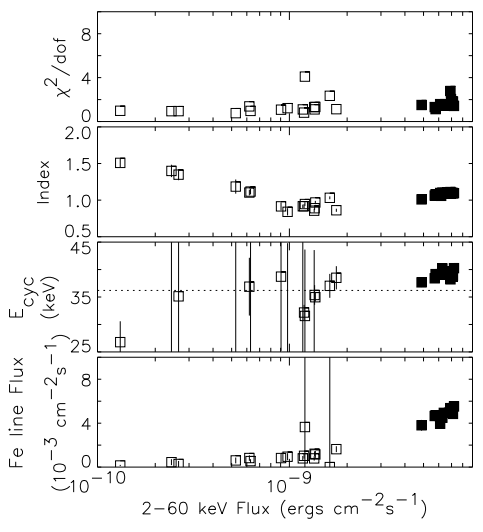

Fig. 8.- Spectral fitting results for an absorbed power law with a cyclotron absorption feature and a Gaussian iron line. Shown are the goodness of fit $\chi^{2} /$ d.o.f., the power law photon index, the cyclotron line energy $E_{\mathrm{cyc}}$, and the integrated flux in the iron line versus $2-60 \mathrm{keV}$ flux. The dotted line denotes the cyclotron energy measured during the 1998 outburst by Heindl et al. (2002). Filled squares denote the 1998 outburst and open squares denote the last pair of outbursts observed in 2001.

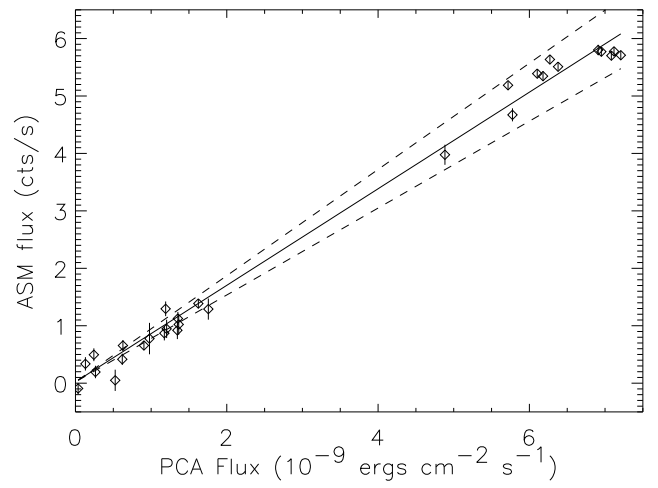

Fig. 9.- ASM count rate vs. PCA 2-60 keV flux measurements. The solid line denotes our best fit the the correlation. This fit was used to estimate a bolometric correction to the ASM data. Dashed lines correspond to our best fit slope $\pm 10 \%$ indicating our estimate of systematic error due to changing beaming angles at high fluxes. 


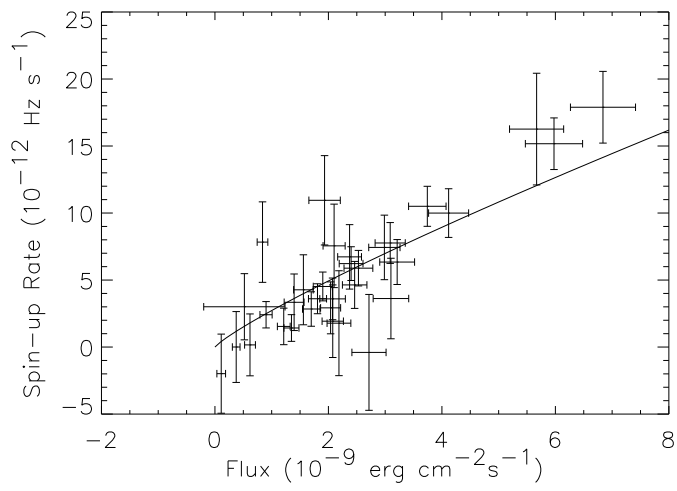

Fig. 10. - Spin-up rate vs. bolometrically corrected ASM flux. The solid line is our best fit of the simple torque model with a distance of $9.5 \mathrm{kpc}$.
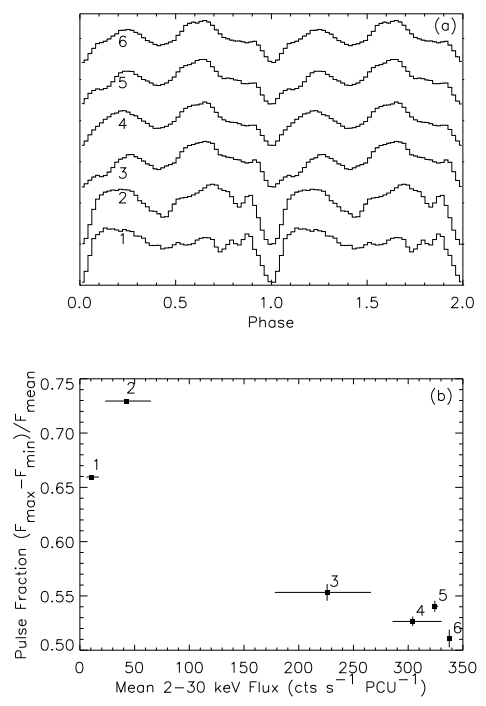

Fig. 11.- (a): Average 2-30 keV pulse profiles from 6 intensity bands versus intensity. Profiles are numbered 1-6, corresponding to descriptions in the main text. Profiles 1 and 2 are from the 2001 outbursts. Profiles 4 and 5 are from similar intensities during the rise and fall, respectively, of the 1998 outburst. Each profile has been normalized to its mean flux. Profiles are offset by arbitrary amounts to keep them from overlapping. (b): Peak-to-peak pulse fraction versus mean 2-30 $\mathrm{keV}$ flux for the pulse profiles shown in panel (a). Horizontal bars indicate the flux range spanned by the profiles included in the average.

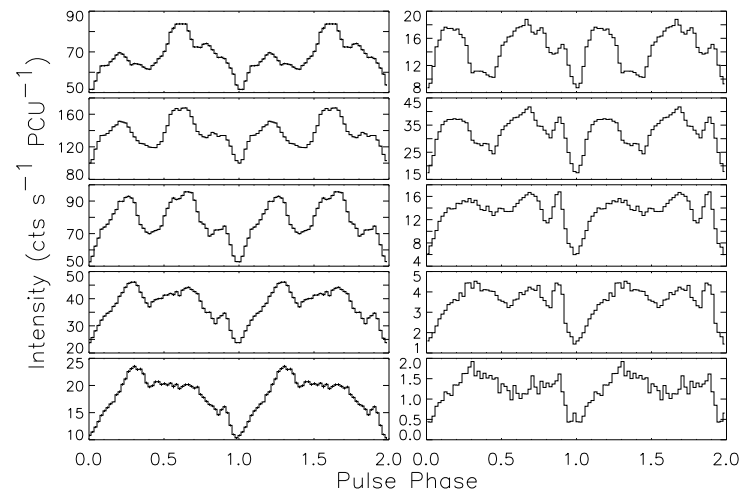

Fig. 12.- Pulse profiles in 5 energy bands (from top to bottom) $2-5,5-10,10-15,15-20,20-30 \mathrm{keV}$ from the peak of the 1998 outburst (left) and the 2001 outbursts (right).

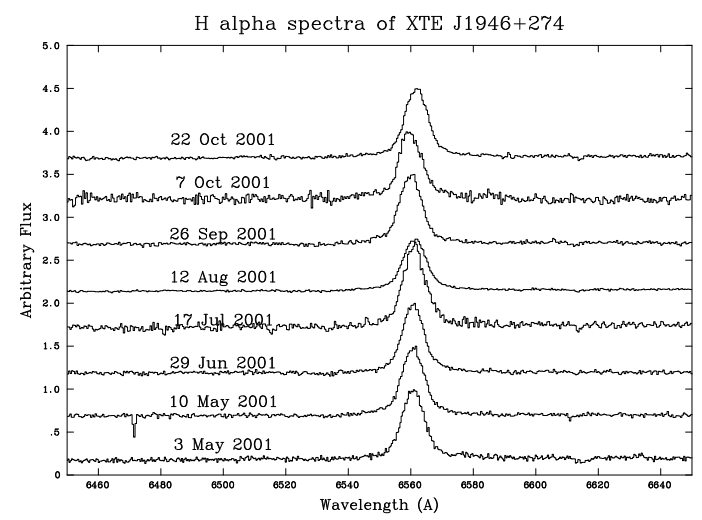

Fig. 13. - $\mathrm{H} \alpha$ profiles from 7 observations of XTE J1946+274 during our 2001 X-ray observations. The small dip near $6470 \AA$ in the 10 May 2001 spectrum is most likely due to a cosmic ray hit. 


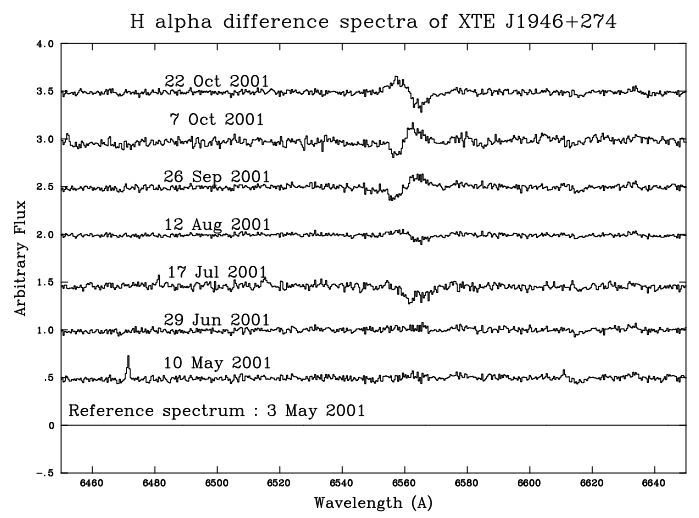

Fig. 14.- $\mathrm{H} \alpha$ profiles in Figure 13 were normalized to the same peak value and then the profile from 2001 May 3 was subtracted from all other profiles to illustrate differences in the profiles. At some time between 2001 Jun 29 and Jul 17, the profile began to change rapidly.

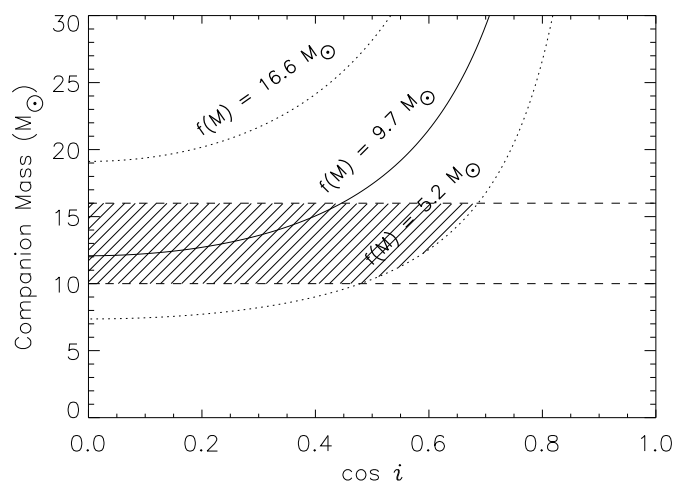

Fig. 15.- Companion Mass versus the cosine of the inclination angle. The solid line is the mass function from the fourth column of Table 2. Dotted lines denote the $68 \%$ confidence error region on the mass function. Dashed lines indicate the range of companion masses derived from optical observations. The hatched region denotes the allowed masses and inclinations. 